\title{
Une géographie des vulnérabilites territoriales face aux risques zoonotiques émergents
}

Héloïse Lucaccioni

\section{OpenEdition}

Journals

Édition électronique

URL : http://journals.openedition.org/rfst/613

DOI : $10.4000 /$ rfst.613

ISSN : 2492-3672

\section{Éditeur}

Espaces et SOciétés (UMR 6590)

\section{Référence électronique}

Héloïse Lucaccioni, «Une géographie des vulnérabilites territoriales face aux risques zoonotiques émergents », Revue francophone sur la santé et les territoires [En ligne], Ateliers et séminaires, mis en ligne le 25 novembre 2016, consulté le 06 avril 2021. URL : http://journals.openedition.org/rfst/613 DOI : https://doi.org/10.4000/rfst.613

Ce document a été généré automatiquement le 6 avril 2021.

\section{(i) (2)}

La Revue francophone sur la santé et les territoires est mise à disposition selon les termes de la Licence Creative Commons Attribution - Pas d'Utilisation Commerciale - Partage dans les Mêmes Conditions 4.0 International. 


\title{
Une géographie des vulnérabilites territoriales face aux risques zoonotiques émergents
}

\author{
Héloïse Lucaccioni
}

1 Dans cette contribution, je présente mes travaux de thèse qui portent sur les dynamiques d'invasion d'un hôte de zoonose dans un territoire en mutation, à travers l'exemple du rat noir au Sénégal Oriental. J'y expose la problématique et les objectifs de cette recherche, le cadre théorique et méthodologique, puis les grandes étapes de la démonstration. À travers ces trois parties, je m'attache à montrer l'originalité de ma recherche et son insertion parmi les innovations et la variété des approches en géographie de la santé.

\section{Espaces, liens, et santé : problématique et objectif de la recherche}

2 Dans ma thèse de doctorat, je dresse une géographie des vulnérabilités territoriales face aux risques zoonotiques émergents. Cette recherche répond à des enjeux relatifs aux émergences infectieuses contemporaines face aux transformations des espaces et des sociétés, qui conjuguent changements globaux et intensification des relations spatiales (Armelagos 1988; Barrett et Armelagos 2013 ; Barrett et al 1998; Louria 2000 ; Mc Michael 2004; Morse 1995). En particulier, ma problématique renvoie à des préoccupations quant à l'expansion des aires de répartition d'espèces réservoirs et au risque associé d'émergence de zoonoses.

3 J'illustre cette problématique à travers les dynamiques d'invasion d'un hôte invasif, le rat noir (Aplin et al 2011; Battersby 2015; Meerburg et al 2009), dans une périphérie rurale en mutation d'Afrique de l'Ouest, le Sénégal Oriental.

4 Le positionnement du géographe sur cet objet a priori biomédical à l'interface des relations environnements-sociétés soulève un enjeu majeur: il s'agit de s'affranchir 
d'une approche centrée sur les mécanismes épidémiologiques et leurs facteurs de risque pour s'interroger sur le rôle des transformations des espaces et des sociétés dans les dynamiques spatio-temporelles du risque. En particulier, je m'interroge sur l'évolution des espaces et des liens et leur rôle dans les dynamiques d'invasion du rat noir. Je défends l'idée que les dynamiques (spatio-temporelles) du risque de zoonoses porté par le rongeur s'inscrivent dans des systèmes territoriaux complexes et multiscalaires, et je cherche à comprendre la construction, par les sociétés, de ces territoires vulnérables.

5 Cette recherche s'inscrit donc dans une problématique large qui croise le risque d'émergence infectieuse, les invasions biologiques, et les dynamiques territoriales à l'échelle du sud-est sénégalais. Au sein de cette problématique pluridisciplinaire, j'élabore des questionnements géographiques spécifiques: je souhaite analyser les interrelations entre les mises en liens et les transformations des espaces et des sociétés du sud-est sénégalais comme producteurs de vulnérabilité territoriale face à la diffusion spatiale du rat noir. Cette recherche revêt ainsi une dimension théorique et préventive face au risque de zoonoses portées par le rat noir.

\section{Quelle géographie pour étudier les dynamiques spatio- temporelles du risque de zoonoses?}

6 Cette recherche s'inscrit au croisement d'une géographie des maladies et d'une géographie nomothétique sur les processus de diffusion spatiale.

7 Avant tout, nos travaux s'approprient l'héritage d'une géographie des maladies d'inspiration francophone, dans la lignée des travaux de Max Sorre. Cette géographie sous-tend l'idée générale que la réunion de certaines conditions (biologiques, sociales, etc.) permet de délimiter l'espace du risque infectieux. Les travaux des géographes contemporains ont continué d'explorer les interrelations entre espaces et risque infectieux en se détachant du déterminisme du milieu et en développant une approche plus systémique. Ce sont les modes de gestion de l'environnement, les pratiques spatiales des sociétés, ainsi que les modes de contrôle et de gestion des territoires qui façonnent les disparités spatiales des faits infectieux (Hervouët et al 1995; Handschumacher 2011 ; Salem 1998).

En parallèle, les dynamiques spatio-temporelles du risque renvoient au «mégaconcept » (Raffestin 1984) de la diffusion spatiale. Les travaux des géographes sur les phénomènes de diffusion s'inscrivent avant tout dans une géographie nomothétique, aux références majoritairement anglophones (par exemple, Pyle, Gould, Haggett, Cliff, etc.). Depuis les travaux précurseurs de Hägerstrand (1967), les géographes se sont efforcés d'élaborer un cadre théorique de ces processus spatio-temporels. En cherchant à relier l'organisation de l'espace des sociétés et les formes de la diffusion, ils mettent en évidence l'existence de régularités des formes spatiales de la diffusion et développent des modèles (vagues de diffusion, diffusion hiérarchique, diffusion par contagion, etc.).

9 Nous argumentons qu'un des enjeux principaux de la compréhension des phénomènes de diffusion est de réconcilier les formes spatiales et les processus sociaux explicatifs de ces dernières. Autrement dit, nous pensons que les phénomènes de diffusion spatiale révèlent l'organisation de l'espace au sens structurel (par exemple, la hiérarchie des 
lieux, leur connectivité, leur distance spatiale, etc.), mais également le fonctionnement des sociétés qui produisent cet espace (par exemple, par la diversité des processus sociaux, économiques, politiques, etc.).

10 En appliquant ce cadre méthodologique à notre problématique, nous faisons du rat noir un objet pour faire de la géographie : les dynamiques d'invasion du rongeur deviennent des marqueurs des dynamiques socio-spatiales. Ainsi, lire les vulnérabilités territoriales aux risques zoonotiques émergents portés par le rat noir en tant que phénomène de diffusion spatiale c'est avant tout chercher à comprendre comment les sociétés participent aux dynamiques spatio-temporelles d'invasion du rongeur.

\section{Vulnérabilités territoriales au risque zoonotique émergent : quelques éléments de la démonstration}

11 Notre recherche s'appuie sur le postulat selon lequel la progression du rat noir dépend essentiellement des transports offerts par l'homme. Au Sénégal, des travaux précédents (Duplantier et al 1991; Konečný et al 2013) ont montré que la progression du rongeur depuis le XVème siècle s'est réalisée depuis les comptoirs coloniaux atlantiques jusqu'à l'intérieur des terres en s'appuyant sur les voies fluviales, les voies ferroviaires, et plus tardivement le réseau routier, supports des circulations de biens et de personnes.

Dans le Sud-Est du Sénégal, le rongeur atteint les marges de son aire de répartition. Il s'y étend à la faveur de nouvelles mises en liens à la fin des années 1990. En particulier, il est capturé pour la première fois dans l'extrême Sud un an après l'ouverture de la route bitumée qui relie les deux capitales régionales (Tambacounda et Kédougou) (Bâ 2002). Or le Sénégal Oriental est un territoire en mutation, une périphérie rurale qui s'ouvre sous l'influence de dynamiques historiques et contemporaines (attributions de diverses vocations agricoles, développement des corridors routiers internationaux, des activités extractives, etc.).

Pour comprendre le lien entre l'évolution des relations spatiales et les transformations des espaces, et les vulnérabilités territoriales à la diffusion du rongeur, notre recherche consiste en trois grandes étapes. Premièrement, nous dressons un diagnostic des dynamiques territoriales du Sénégal Oriental. Il s'agit d'une approche avant tout qualitative qui s'appuie sur la littérature scientifique et grise. Nous décrivons des espaces, des lieux, et des liens singuliers comme le produit d'héritages et de recompositions par les multiples opérateurs territoriaux : les disparités des espaces et des liens témoignent d'un encadrement territorial inégal selon les projets politiques et économiques des acteurs territoriaux successifs. En parallèle, nous réalisons un diagnostic de l'invasion du rongeur à partir de données d'archives et récentes de piégeages (Duplantier et al 2009). Nous montrons que la distribution du rat noir varie dans l'espace.

Dans une seconde étape, nous cherchons à établir une relation entre les variabilités spatiales et la distribution du rongeur. À l'aide de statistiques exploratoires, nous construisons des typologies des lieux à partir des caractéristiques locales susceptibles de conditionner leurs mises en liens (activités commerciales, connectivité au réseau routier et de transport, etc.). Pour cela, nous avons recours à des données primaires récoltées sur le terrain. Nous testons ensuite statistiquement des hypothèses sur la distribution du rat noir selon les types de lieux identifiés. Pour compléter cette analyse, 
nous dressons un portrait des mises en liens de ces lieux selon une approche qualitative. Autrement dit, nous explicitons la nature des relations spatiales (commerciales, agricoles, etc.) et leurs dynamiques selon les types de lieux.

Nos analyses aboutissent à un paradoxe : nous révélons une hiérarchie des lieux telle que les facteurs de vulnérabilité (caractéristiques de centralité, connectivité au réseau routier et de transport, etc.) apparaissent flous et complexes. En outre, ni cette hiérarchie des lieux, ni la distance spatiale ne permettent d'expliquer la diffusion du rongeur et sa répartition actuelle parmi les localités du Sénégal Oriental. En effet, le rat noir est présent là où on s'y attend le moins : dans les localités des marges agricoles les plus isolées au détriment des pôles commerciaux du goudron. Nous proposons alors de réinscrire la diffusion spatiale du rongeur en considérant non plus les lieux ou les liens seuls, mais les systèmes territoriaux.

Dans une dernière étape, nous réinterprétons la hiérarchie des lieux en dépassant les critères classiques d'analyse spatiale et des grilles de lecture structurelle des espaces. Nous proposons ainsi de comprendre les dynamiques d'invasion du rat noir dans l'articulation à plusieurs échelles des espaces, des lieux, et des liens. En cela, nous cherchons à réconcilier l'approche structurelle de l'organisation spatiale et le fonctionnement de ces territoires. Les systèmes territoriaux décrits apparaissent plus ou moins stables et porteur de différentes potentialités en matière de diffusion du rat. Combinés avec des systèmes bioécologiques, ils éclairent la répartition actuelle du rongeur dans les marges sud-est du Sénégal.

17 À travers des méthodes mixtes et un cadre méthodologique au croisement d'une posture constructiviste et nomothétique, nous dressons une géographie des vulnérabilités territoriales au risque zoonotique émergent incarné par le rat noir. En étudiant les dynamiques d'invasion du rat noir dans cette périphérie en transformation, je montre que nous ne sommes pas uniquement confrontés à des lienssupports mais à des systèmes sociaux et spatiaux qui articulent espaces, lieux, et liens.

\section{BIBLIOGRAPHIE}

APLIN K.P., SUZUKI H., CHINEN A.A., CHESSER R.T., TEN HAVE J., DONNELLAN S.C., AUSTIN J., FROST A., GONZALES J.-P., HERBRETEAU V., CATZEFLIS F., SOUBRIER J., et al., 2011. Multiple Geographic Origins of Commensalism and Complex Dispersal History of Black Rats. PloS One. Vol. 6, nº11, p. e26357. DOI 10.1371/journal.pone.0026357.

ARMELAGOS G.J., 1998. The Viral Superhighway. The Sciences. Vol. 38, nº1, p. 24-29. DOI 10.1002/j. 2326-1951.1998.tb03354.x.

BÂ, 2002. Systématique, écologie et dynamique de populations de petits rongeurs potentiellement réservoirs ou hôtes de virus au Sénégal. Mémoire du diplôme de Sciences de la Vie et de la Terre de l'Ecole Pratique des Hautes Etudes. Montpellier, France : EPHE, IRD. 132 p.

BARRETT R., ARMELAGOS G., 2013. An Unnatural History of Emerging Infections. Oxford : Oxford University Press. 160 p. ISBN 978-0-19-960829-4. 
BARRETT R., KUZAWA C.W., MC DADE T., ARMELAGOS G.J., 1998. Emerging and Re-Emerging Infectious Diseases: The Third Epidemiologic Transition. Annual Review of Anthropology. Vol. 27, p. 247-271. DOI 10.2307/223371.

BATTERSBY S.A., 2015. Rodents as carriers of disease. In BUCKLE A.P., SMITH R.H. (éd.), Rodent Pests and their Control. 2nd ed. Oxford : CABI. p. 81-100. ISBN 978-1-84593-817-8.

DUPLANTIER J.-M., GRANJON L., ADAM F., BA K., 1991. Répartition actuelle du rat noir (Rattus rattus) au Sénégal : facteurs historiques et écologiques. In LE BERRE M., LE GUELTE L. (éd.), Le rongeur et l'espace. Paris : R. Chabaud. p. 339-346.

DUPLANTIER J.-M., GRANJON L., LE FUR J., PIRY S. (éd.), 2009. Base de Données sur les Rongeurs Sahélo-Soudaniens. CBGP, UMR (Inra/IRD/Cirad/Montpellier SupAgro).

HÄGERSTRAND T., 1967. Innovation Diffusion as a Spatial Process. Chicago, USA: University of Chicago Press. 334 p. ISBN 978-0-226-31261-3.

HERVOUËT J.-P., HANDSCHUMACHER P., LAFFLY D., 2004. Mobilités et espaces partagés au centre du risque sanitaire: l'exemple des endémies tropicales à transmission vectorielle. In GILBERT D.

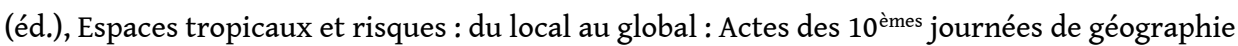
tropicale, 24-26 septembre 2003. Orléans, France : Presses Universitaires d'Orléans. p. 127-158.

HERVOUËT J.-P., HANDSCHUMACHER P., PARIS F., SALEM G., 1995. De la géographie des grandes endémies à la géographie de la santé et à la géographie tout court : vingt ans de travaux de l'ORSTOM en Afrique. Espace, populations, sociétés. Vol. 13, nº1, p. 59-65. DOI 10.3406/espos. 1995.1671.

HANDSCHUMACHER P., 2011. Dynamique des espaces à risques sanitaires dans les pays du Sud : des systèmes entre tropicalité et mal-développement. In BART F. (éd.), Natures tropicales : enjeux actuels et perspectives. Bordeaux, France : Presses Universitaires Bordeaux. ISBN 978-286781729.

KONEČNÝ A., ESTOUP A., DUPLANTIER J.-M., BRYJA J., BA K., GALAN M., TATARD C., COSSON J.-F., 2013. Invasion genetics of the introduced black rat (Rattus rattus) in Senegal, West Africa. Molecular Ecology. Vol. 22, n², p. 286-300. DOI 10.1111/mec.12112.

LOURIA D.B., 2000. Emerging and re-emerging infections: the societal determinants. Futures. Vol. $32, n^{\circ} 6$, p. 581-594

MC MICHAEL A.J., 2004. Environmental and social influences on emerging infectious diseases: past, present and future. Philosophical Transactions of the Royal Society of London. Series B: Biological Sciences. Vol. 359, nº1447, p. 1049-1058. DOI 10.1098/rstb.2004.1480.

MEERBURG B.G., SINGLETON G.R., KIJLSTRA A., 2009. Rodent-borne diseases and their risks for public health. Critical Reviews in Microbiology. Vol. 35, n³, p. 221-270. DOI 10.1080/10408410902989837.

MORSE S.S., 1995. Factors in the emergence of infectious diseases. Emerging infectious diseases. Vol. 1, nº1, p. 7-15. DOI 10.3201/eid0101.950102.

RAFFESTIN C., 1984. La diffusion. In BAILLY A. (éd.), Les concepts de la géographie humaine. Paris, France : Masson. p. 189-193. ISBN 978-2-225-80290-4.

SALEM G., 1998. La santé dans la ville : géographie d'un petit espace dense : Pikine (Sénégal). Paris, France : Karthala, ORSTOM. 360 p. ISBN 2-86537-826-8.

SORRE M., 1933. Complexes pathogènes et géographie médicale. Annales de Géographie. Vol. 42, n²35, p. 1-18. DOI 10.3406/geo.1933.10619. 
INDEX

Index géographique : Sénégal

Mots-clés : zoonose, vulnérabilités, risque, transformations des espaces et des sociétés

AUTEUR

HÉLOÏSE LUCACCIONI

Laboratoire Dynamiques Sociales et Recomposition des Espaces (LADYSS), Université Paris Ouest Nanterre La Défense 